\title{
Real-Time SVD for the Control of Redundant Robotic Manipulators
}

Anthony A. Maciejewski

School of Electrical Engineering

Purdue University

West Lafayette, IN 47907

\begin{abstract}
By taking advantage of the well-behaved nature of singular values and vectors, the computational expense of determining the SVD of the Jacobian matrix can be reduced to such an extent that on-line calculation becomes feasible. This permits more intelligent use of the extra degrees of freedom present with redundant manipulators, particularly with respect to the optimization of various secondary criteria, including obstacle avoidance, under the constraint of a specified end effector trajectory.
\end{abstract}

\section{Introduction}

In recent years the singular value decomposition (SVD) has become a popular tool for analyzing the kinematic and dynamic properties of robotic manipulators. It plays a particularly prominent role with regard to redundant manipulators, both in term of analyzing the significance of the extra degrees of freedom [2] and in specifying a side criterion which can be optimized using these redundant degrees of freedom. In many cases, these side criteria are some quantitative measure of the qualitative concept of dexterity. Most of the dexterity measures proposed are some function of the singular values of the Jacobian matrix. The most common of these is perhaps the manipulability measure proposed by Yoshikawa [12] which is defined as the square root of the determinant of the matrix $J J^{T}$ which is simply the product of the singular values of $J$. Other proposed measures include the trace of the above matrix, the minimum singular value of the Jacobian, the compatibility index, and isotropy (all equal singular values).

While all of the above measures have a physical significance and justification for their use, the key point here is that they are all closely linked to the SVD. Yet in spite of this fact, the full decomposition is usually limited to the analysis of manipulator configurations and is not considered for implementation in on-line control. This work demonstrates that, with the right formulation, the SVD is computationally feasible for use in real-time control. Traditionally, the computation of the SVD of an arbitrary matrix is an iterative procedure so that the exact number of computations cannot be known $a$ priori.
However, the control of robotic systems is not based on the solution of arbitrary matrix equations but quite frequently involves the solution of equations based on the Jacobian matrix. The current Jacobian for a system can be regarded as a perturbation of a previously known matrix for which perturbation bounds on the singular values and singular vectors can be established. Knowledge of the previous state can be exploited during the current calculation of the SVD in order to reduce the overall computational burden. This results in a computational scheme capable of calculating the SVD of the Jacobian for use in real-time control of manipulators.

\section{Perturbation Bounds on the SVD}

All SVD algorithms possess an iterative component designed to orthogonalize the columns (or rows) of the matrix being decomposed. Clearly, the more orthogonal these columns are to begin with, the fewer the number of calculations are required to reach convergence. Thus, if one considers the current manipulator Jacobian, denoted by $J(t+\Delta t)$, to be a perturbation of the previous Jacobian

$$
J(t+\Delta t)=J(t)+\Delta J(t)
$$

the SVD of which is known and given by

$$
J(t)=U(t) D(t) V^{T}(t)
$$

then the matrix $J(t+\Delta t) V(t)$ will have nearly orthogonal columns provided the perturbation $\Delta J(t)$ is small relative to $J(t)$. The foundation of the above lies in the fundamentally well-behaved nature of the SVD of a matrix. The perturbation bounds on singular values, denoted by $\sigma_{i}$, are very well-known and it is easy to show that

$$
\left|\sigma_{i}(J(t+\Delta t))-\sigma_{i}(J(t))\right| \leq\|\Delta J(t)\| .
$$

The perturbation bounds on the rotation of subspaces defined by singular vectors are not as widely known but are also well-behaved [11]. In particular, consider a partitioning of the Jacobian into two singular subspaces of the form

$$
J=J_{1}+J_{0}=\sum_{i=1}^{k} \sigma_{i} u_{i} v_{i}^{T}+\sum_{i=k+1}^{m} \sigma_{i} u_{i} v_{i}^{T}
$$


where $u_{i}$ and $v_{i}$ are the columns of $U$ and $V$, respectively, and the integer $k$ denotes the partitioning between the large and the small singular values. The angle between the subspace $J_{0}(t)$ and its perturbation $J_{0}(t+\Delta t)$ can be defined as

$$
\left\|\sin \phi\left(J_{0}(t), J_{0}(t+\Delta t)\right)\right\|=\left\|\left(I-P_{J_{0}(t+\Delta t)}\right) P_{J_{0}(t)}\right\|
$$

where $P_{J_{0}}$ denotes the orthogonal projection onto the subspace $J_{0}$. With the above definition, an upper bound on $\phi$ will guarantee that the subspaces defined by the singular vectors will change in a well-defined manner.

The bounds for rotations of subspaces are closely related to the spacing of the $k$ th and the $k+1$ th singular values. In particular, if the gap between $\sigma_{k}$ of $J(t+\Delta t)$ and $\sigma_{k+1}$ of $J(t)$ is denoted by $\delta$ then it can be shown [11] that the bound on subspace rotation is given by

$$
\left\|\sin \phi\left(J_{0}(t+\Delta t), J_{0}(t)\right)\right\|=\sqrt{2} \frac{\|\Delta J(t)\|}{\delta} .
$$

The above bound guarantees continuity of the singular vectors as long as $\delta$ does not approach zero. Geometrically, what occurs when $\delta \rightarrow 0$ is that there are multiple equal singular values. When this occurs the singular vectors are ill-defined since the subspace associated with the corresponding singular values is no longer one dimensional and any orthogonal set of vectors which spans this subspace is a valid set of singular vectors.

\section{Calculation of the SVD}

The Golub-Reinsch algorithm is generally regarded as the most efficient and numerically stable technique for computing the SVD of an arbitrary matrix. However, an algorithm based exclusively on Givens rotations is more suited to take advantage of the incremental perturbations of the Jacobian matrix. The basis of this algorithm is the ability of a single Givens rotation to orthogonalize any two columns on which it operates. In particular, if $a_{i}$ and $a_{j}$ are denoted as the $i$ th and $j$ th columns of the matrix $A$, then multiplication by a Givens rotation results in the new columns, $a_{i}^{\prime}$ and $a_{j}^{\prime}$ given by

$$
\begin{aligned}
& a_{i}^{\prime}=a_{i} \cos (\theta)+a_{j} \sin (\theta) \\
& a_{j}^{\prime}=a_{j} \cos (\theta)-a_{i} \sin (\theta) .
\end{aligned}
$$

It can be easily shown that one can choose the rotation angle $\theta$ such that $a_{i}$ and $a_{j}$ are orthogonal. To orthogonalize all of the columns, Givens rotations are applied to all combinations of pairs of columns, a process referred to as a sweep. Unfortunately, a single sweep will not, in general, orthogonalize all of the columns of a matrix since subsequent rotations can destroy the orthogonality produced by previous ones, however, the procedure can be shown to converge.

While the number of sweeps required to orthogonalize the columns of the Jacobian cannot, in general, be determined a priori it should be clear that the more orthogonal the columns are to begin with, the fewer the number of sweeps required. Simulation results show that by using the knowledge of the previous SVD as discussed in section II above, the number of sweeps can be cut by a factor of three. In addition, it can be shown that in many cases a single sweep is sufficient so that convergence tests can be removed from the computation. A further reduction in the computational complexity of the equations for computing the SVD can be gained by noting that the majority of the rotations are very small in magnitude. As an example, consider a typical trajectory for a PUMA robot, presented in Fig. 1, which illustrates the singular values of the Jacobian for each configuration of the trajectory along with the maximum rotation angle required for orthogonalization. Note that for the majority of the trajectory the maximum rotation angle is very small, however, there exist three peaks, two of which are very sharp. From comparing the position of these peaks to the spacing of the singular values, it is clear that very large rotations are required when there is a crossing of adjacent singular values. This, of course, is exactly what is predicted by the perturbation bounds on the rotation

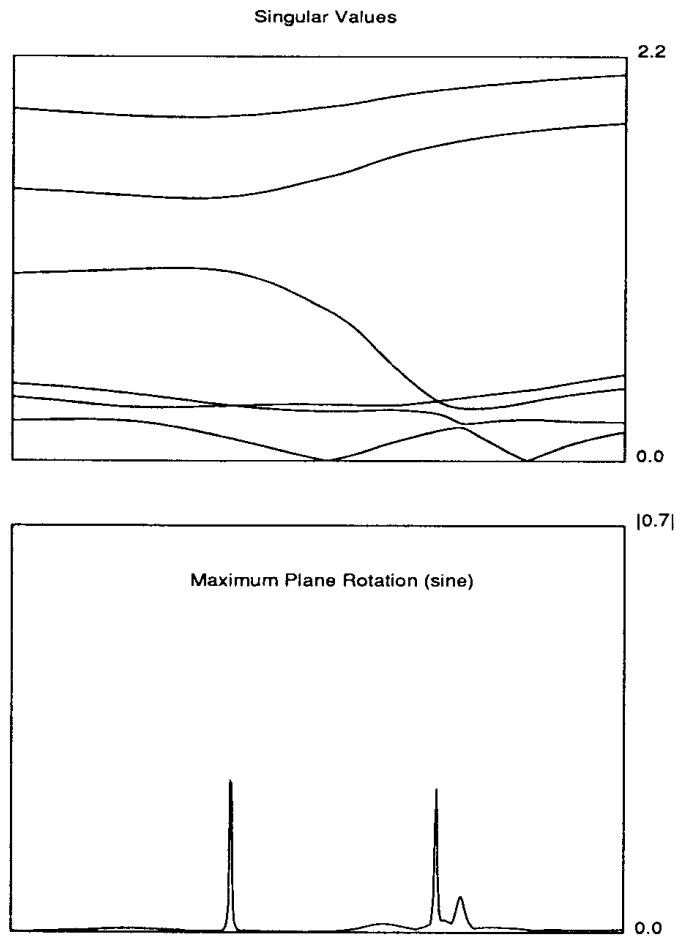

Fig. 1 The singular values of the Jacobian for each point of a typical trajectory along with the maximum magnitude of the plane rotation required during the computation of the SVD. 
of subspaces in section II. Since the maximum plane rotations required during the algorithm are very small in magnitude outside of a few isolated peaks, a small angle approximation is useful in reducing the computational effort required in computing the SVD. For small $\theta$ the approximations $\cos (\theta) \approx 1$ and $\sin (\theta) \approx \theta$ can be used so that the number of multiplications required in calculating the rotation of (7) and (8) is cut in half. Those configurations for which the small angle approximation is not valid are easily identified by examining the spacing of the singular values.

In terms of actual CPU time, an algorithm using the previous estimate of the SVD as well as the small angle approximations was implemented in PASCAL and executed on a VAX785. The time required to calculate the SVD of the Jacobian for the PUMA manipulator used in Fig. 1 was 5.13 ms. By comparison, the Golub-Reinsch algorithm in the IMSL package required an average of $32.3 \mathrm{~ms}$. Details of the algorithm and its implementation can be found in [7].

\section{Control of Redundant Manipulators}

Real-time computation of the SVD can improve the utilization of redundancy in robotic systems both in terms of implementation of the control equations as well as in the evaluation of various measures of dexterity. The kinematics of manipulators is frequently presented in the form

$$
J \dot{\theta}=\dot{x}
$$

where $\dot{x}$ is an $m$ dimensional vector specifying the end effector velocity, $\dot{\theta}$ is an $n$ dimensional vector denoting the joint velocities, and $J$ is the $m$ by $n$ Jacobian matrix. For redundant manipulators $n>m$ so that the general solution to (9) is typically presented in the form

$$
\dot{\theta}=J^{+} \dot{x}+\left(I-J^{+} J\right) \dot{\phi}
$$

where ${ }^{+}$denotes the pseudoinverse, $\left(I-J^{+} J\right)$ is a projection operator onto the null space of $J$, and $\dot{\phi}$ is an arbitrary vector in $\dot{\theta}$ space. The second term in (10) is the homogeneous solution to (9) since it results in no end effector velocity. This homogeneous solution is frequently used to optimize some secondary criterion under the constraint of the specified end effector velocity by choosing $\dot{\phi}$ to be the gradient of some function of $\theta[3]$. Some of the secondary criteria that have been applied include joint range availability, singularity avoidance, various measures of dexterity, and obstacle avoidance. With the complete SVD available the projection operation becomes trivial since the singular vectors $v_{i}$ for $r>i \geq n$ specify an orthonormal basis for the null space. Thus the relative advantages of using the homogeneous solution for alternate secondary criteria can be easily evaluated.

The homogeneous solution can also be used to optimize secondary criteria defined in Cartesian space, either to impose a priority to the manipulation variables [9] or to avoid obstacles [5], by using

$$
\dot{\phi}=\left[J_{2}\left(I-J^{+} J\right)\right]^{+}\left(\dot{x}_{2}-J_{2} J^{+} \dot{x}\right)
$$

where the subscript 2 refers to the secondary criterion. The overall solution is then given by substituting (11) into (10) to obtain

$$
\dot{\theta}=J^{+} \dot{x}+\left[J_{2}\left(I-J^{+} J\right)\right]^{+}\left(\dot{x}_{2}-J_{2} J^{+} \dot{x}\right)
$$

which has been simplified by taking advantage of the fact that the projection operator is both Hermetian and idempotent [5]. In this case it is also desirable to obtain the SVD of the matrix $J_{2}\left(I-J^{+} J\right)$. The same algorithm performed on the original Jacobian can be applied to this matrix along with the additional information that a significant number of the singular vectors are already known. In fact, since one is guaranteed that the range of the singular vectors associated with $J_{2}\left(I-J^{+} J\right)$ is limited to the null space of $J$, as shown in [5], the singular vectors $v_{1}$ to $v_{r}$ of $J$ are also singular vectors of $J_{2}\left(I-J^{+} J\right)$. This knowledge significantly reduces the calculations required for computing the complete SVD of $J_{2}\left(I-J^{+} J\right)$. A similar scenario occurs in the use of redundancy for torque minimization [1].

\section{Dealing with Singularities}

Singular configurations can be identified by a mathematical change of rank in $J$ which physically represents the inability of the manipulator to achieve an arbitrary end effector velocity. A general approach to resolving the discontinuity at singular configurations and maintaining a well-conditioned formulation which results in physically meaningful joint velocities is to use the damped least-squares formulation independently proposed in [8] and [10] and extended in [6]. The damped least squares solution of (9), denoted by $\dot{\theta}^{(\lambda)}$, is the solution which minimizes the sum $\|\dot{x}-J \dot{\theta}\|+\lambda\|\dot{\theta}\|$. Unfortunately, the norm of the solution is a nonlinear function of the damping factor, $\lambda$, so that iterative techniques such as Newton's method must be applied to determined the optimal damping factor for a given constraint on the physically achievable joint velocity norm.

While the damped least-squares solution is optimal in the sense described above, it may be undesirable to implement due to the iterative nature of calculating the appropriate damping factor $\lambda$. It can be shown that the characteristics of the damped least-squares solution are very similar to those obtained using a continuous version of the truncated SVD solution. This type of solution will be denoted $\dot{\theta}^{(\mathrm{c})}$ and is defined by

$$
\dot{\theta}^{(c)}=\sum_{i=1}^{k} \frac{\dot{x}_{i}}{\sigma_{i}} v_{i}+\frac{(c-k) \dot{x}_{k+1}}{\sigma_{k+1}} v_{k+1}
$$

where $c$ is a real number less than or equal to the rank and $k$ is the greatest integer less than or equal to $c$. The 


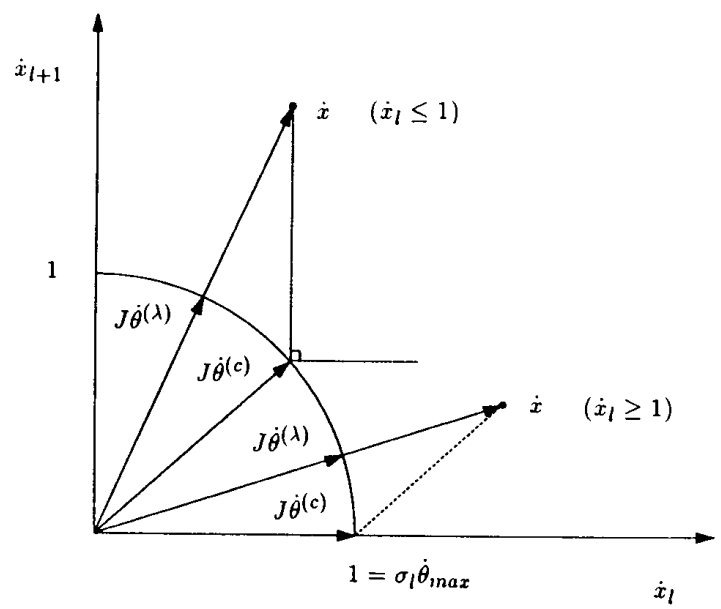

Fig. 2 A comparison of the residual error of the damped least-squares solution and the continuous truncated SVD solution in a worst-case scenario.

advantages of using this form of a solution are that, when the SVD is available, it is extremely easy to compute.

By performing a worst case analysis, it can be shown [4] that the error in the continuous truncated SVD solution can be as much as $40 \%$ greater than the damped least-squares solution. While a $40 \%$ discrepancy may at first seem as cause for concern, it can be shown that this case will occur only under very special circumstances. In particular, analysis shows that it will occur only if the manipulator is in a configuration which results in two or more very small and equal singular values. That is, the manipulator must be going into a multiple singularity. In addition, the commanded end effector velocity must be restricted to these two or more directions which represent the lost degrees of freedom. Finally, the magnitude of this commanded end effector velocity must be on the order of magnitude of the very small singular values. An example of this condition illustrating the plane defined by the singular vectors associated with the two equal and very small singular values is presented in Fig. 2. Since these conditions are very restrictive and easily identified when the SVD is available, the more accurate damped least-squares solution can be employed if desired. In these cases, the value of $c$ provides a good starting point for the Newton iteration scheme.

\section{Conclusions}

It has been shown that the incremental manner in which the Jacobian changes, along with the perturbation bounds on singular values and vectors, allows one to reduce the computational expense of calculating the SVD in three ways. First, the singular vectors from the previous calculation can be used to pre-orthogonalize the current Jacobian. Second, by virtue of nearly orthogonal columns, the iterative nature of the calculations can be prevented thus avoiding convergence tests. Finally, since the few rotations required for orthogonalization are small in magnitude, approximations can be used. The advantages of having the SVD available in redundant manipulator control include the optimization of secondary criteria for dexterous operation, obstacle avoidance, and torque minimization as well as the identification of physical limitations corresponding to singular or near-singular configurations.

\section{References}

[1] J. M. Hollerbach and K. C. Suh, "Redundancy resolution of manipulators through torque optimization," IEEE J. Robotics Automation, vol. RA-3, no. 4, pp. 308-316, Aug. 1987.

[2] C. A. Klein and C. H. Huang, "Review of pseudoinverse control for use with kinematically redundant manipular tors," IEEE Trans. on Sys., Man, Cyber., vol. SMC-13, pp. 245-250, March/April 1983.

[3] A. Liegeois, "Automatic supervisory control of the configuration and behavior of multibody mechanisms," IEEE Trans. Sys., Man, Cyber., vol. SMC-7, no. 12, pp. 868-871 Dec. 1977.

[4] A. A. Maciejewski, "The analysis and control of robotic manipulators operating at or near kinematically singular configurations," Ph.D. Thesis, The Ohio State University, 1987.

[5] A. A. Maciejewski and C. A. Klein, "Obstacle avoidance for kinematically redundant manipulators in dynamically varying environments," Int. J. Robotics Res., vol. 4, no. 3, pp. 109-117, Fall 1985.

[6] A. A. Maciejewski and C. A. Klein, "Numerical filtering for the operation of robotic manipulators through kinematically singular configurations," J. Robotic Sys., vol. 5, no. 6, pp. 527-552, Dec. 1988.

[7] A. A. Maciejewski and C. A. Klein, "The singular value decomposition: Its computation and applications to robotics," Int. J. Robotics Res., (in press).

[8] Y. Nakamura and H. Hanafusa, "Inverse kinematic solutions with singularity robustness for robot manipulator control," ASME J. Dyn. Sys., Meas., Contr., vol. 108, no. 3, pp. 163-171, Sept. 1986.

[9] Y. Nakamura, H. Hanafusa, and T. Yoshikawa, "Taskpriority based redundancy control of robot manipulators," Int. J. Robotics Res., vol. 6, no. 2, pp. 3-15, Su 1987.

[10] C. W. Wampler, "Manipulator inverse kinematic solutions based on vector formulations and damped least-squares methods," IEEE Trans. Sys., Man, Cyber., vol. SMC-16, pp. 93-101, Jan./Feb. 1986.

[11] P. A. Wedin, "Perturbation bounds in connection with singular value decomposition," BIT, vol. 12, pp. 99-111, 1972.

[12] T. Yoshikawa, "Manipulability of robotic mechanisms," Int. J. Robotics Res., vol. 4, no. 2, pp. 3-9, Su 1985. 